\title{
ACTAPOL : un chantier de recherche sur les conventionnels
}

Michel Biard, Philippe Bourdin et Hervé Leuwers

\section{(2) OpenEdition \\ 1 Journals}

Édition électronique

URL : https://journals.openedition.org/ahrf/13599

DOI : 10.4000/ahrf.13599

ISSN : 1952-403X

Éditeur :

Armand Colin, Société des études robespierristes

Édition imprimée

Date de publication : 1 septembre 2015

Pagination : 3-9

ISBN : 9782200929855

ISSN : 0003-4436

Référence électronique

Michel Biard, Philippe Bourdin et Hervé Leuwers, «ACTAPOL : un chantier de recherche sur les conventionnels ", Annales historiques de la Révolution française [En ligne], 381 | juillet-septembre 2015, mis en ligne le 01 septembre 2018, consulté le 24 avril 2022. URL : http://journals.openedition.org/ ahrf/13599; DOI : https://doi.org/10.4000/ahrf.13599 


\section{ACTAPOL \\ UN CHANTIER DE RECHERCHE SUR LES CONVENTIONNELS}

Michel BIARD, Philippe BOURDIN et Hervé LEUWERS

Derrière l'acronyme ACTAPOL se cache un projet financé par l'Agence nationale de la recherche, intitulé : « Acteurs et action politique en Révolution. Les conventionnels ». Conçu et porté par des membres des équipes de l'IRHiS (Lille 3, Hervé Leuwers), du GRHis (Rouen, Michel Biard) et du CHEC (Clermont-Ferrand 2, Philippe Bourdin) qui, traditionnellement, consacrent une part notable de leurs travaux aux révolutions du tournant des $\mathrm{XVIII}^{\mathrm{e}}$ et $\mathrm{XIX}^{\mathrm{e}}$ siècles, le programme s'est déployé parallèlement aux projets ANR REVLOI, sur la loi en Révolution, et THEREPSICORE, consacré au théâtre sous la Révolution et l'Empire. Spécifiquement centré sur ce moment éminemment complexe de la fondation (1792-1793), puis de la refondation (1795) républicaine, le programme ACTAPOL s'est donné à cœur d'encourager les recherches sur les conventionnels, leur action et leurs parcours, pendant et après la Première République.

Le cœur du projet est la réalisation d'un dictionnaire biographique des conventionnels, à paraître à Ferney-Voltaire, au Centre international d'étude du XVIII ${ }^{\mathrm{e}}$ siècle, dans la même collection que le Dictionnaire des Législateurs, 1791-1792, publié sous la direction d'Edna Lemay ${ }^{1}$. Ici, l'objectif est de poursuivre l'œuvre de l'historienne, dont les dictionnaires des députés de la Constituante ${ }^{2}$, puis de la Législative, font autorité et rendent d'inappréciables services aux chercheurs. Pour connaître les hommes de

(1) Edna H. LEMAY (dir.), Dictionnaire des Législateurs, 1791-1792, Ferney-Voltaire, Centre international d'étude du XVIII' siècle, 2007, 2 vol.

(2) Idem, Dictionnaire des Constituants, 1789-1791, Paris et Oxford, Universitas et Voltaire Foundation, 1991, 2 vol. 
la troisième Assemblée révolutionnaire, en effet, aucun dictionnaire n'a encore remplacé le classique Dictionnaire des conventionnels d'Auguste Kuscinski, publié en 1916, et réédité en $1973^{3}$. Euvre d'un homme, œuvre d'une vie, ce dictionnaire est demeuré en partie incomplet, l'auteur ayant repoussé la rédaction de la dernière notice, celle de Robespierre, qui est finalement... absente de l'ouvrage édité ; quant aux autres notices, elles rassemblent une documentation extrêmement précieuse, mais assez souvent fautive, faute d'avoir pu toujours être vérifiée sur les sources. De plus, la sympathie affichée par Kuscinski pour les montagnards, accompagnée d'un mépris souvent mal dissimulé pour certains autres conventionnels, a pesé de manière importante sur la longueur, voire la richesse, des notices individuelles. L'objectif est donc de reprendre le dossier, cette fois par un travail collectif rassemblant une cinquantaine de chercheurs et à l'aide de documents difficilement mobilisables par un seul homme : les registres paroissiaux et d'état civil, désormais disponibles en ligne pour la grande majorité des départements ${ }^{4}$, les procès-verbaux d'élection de septembre 1792, les archives de la Convention et de ses comités (fonds C et D des Archives nationales), les sources des clubs et, bien sûr, les débats d'assemblée, qui ont été dépouillés à partir du Moniteur universel et des Archives parlementaires, dont Corinne Gomez-Le Chevanton et Françoise Brunel rappellent, dans ce numéro, l'histoire, les apports et les limites.

En s'inscrivant dans le prolongement des travaux d'Edna Lemay, ce nouveau Dictionnaire des conventionnels proposera une masse d'informations vérifiées sur les origines sociales, la culture et, surtout, le parcours politique des hommes de la Convention. Le cœur des biographies, en effet, sera consacré aux années 1792-1795 pour lesquelles chaque notice précisera successivement : la date et le rang d'élection à la Convention (1), la mouvance ou les mouvances politiques dans lesquelles le représentant du peuple s'inscrit (2), les comités aux travaux desquels il a participé (3), ses autres éventuelles fonctions (président, secrétaire, etc.) au sein de la Convention (4), ses missions comme représentant (5), ses principales interventions à l'Assemblée (6), ses votes aux différents appels nominaux (7), sa participation à la vie des clubs (8), et ses possibles mises en arrestation, en accusation, ou hors de la loi, voire sa mort violente par exécution, suicide, assassinat, ou autre (9). L'ensemble de ces notices livrera un matériau dont

(3) Auguste KuscinsKI, Dictionnaire des conventionnels, Paris, Société de l'histoire de la Révolution française, 1916 ; reprint : Brueil-en-Vexin, Éditions du Vexin français, 1973.

(4) http://www.archivesdefrance.culture.gouv.fr/ressources/en-ligne/etat-civil/ 
la présentation normée permettra des approches prosopographiques, qui ne sont qu'ébauchées à l'issue de notre programme de recherche.

Ces dernières années, pourtant, de premiers matériaux ont pu être analysés. Ces résultats ont été présentés à l'occasion de trois principales rencontres qui sont loin de toutes relever de la prosopographie, tant l'objectif d'ACTAPOL dépasse l'étude des simples parcours d'élus. Le premier colloque, organisé à l'Hôtel de ville de Paris et en Sorbonne, en collaboration avec la Société des études robespierristes et l'Institut d'histoire de la Révolution française de Paris 1, s'est interrogé sur les modalités et les enjeux de l'entrée en République, de la mise en mots du régime nouveau - dans un contexte baigné de la culture de l'Antiquité et des Lumières à l'élection de la Convention et à la fondation du régime nouveau ${ }^{5}$. À l'occasion d'un colloque organisé à l'université Senshu de Tokyo, puis d'une journée d'étude coorganisée avec la Société des études robespierristes, à Ivry-sur-Seine ${ }^{6}$, c'est cette fois la question de la mémoire de l'événement qui a été posée. Enfin, par un colloque organisé à l'Assemblée nationale, et dans une perspective large prenant en compte la vie politique des $\mathrm{XIX}^{\mathrm{e}}$ et $\mathrm{XX}^{\mathrm{e}}$ siècles, ce sont les liens entre la vertu publique et la vie politique qui ont été analysés ${ }^{7}$.

Ce numéro spécial des Annales historiques de la Révolution française se veut un prolongement de ces rencontres. Alors que se termine le programme financé, l'heure n'est pas encore au bilan. Plus modestement, l'objectif est de rassembler neuf contributions qui, par-delà leur diversité, interrogent les sources à la disposition de l'historien. Parmi celles-ci se trouvent les Archives parlementaires, patiemment reconstituées depuis le second Empire et jusqu'à nos jours ; dans leur article, Corinne GomezLe Chevanton et Françoise Brunel rappellent combien leur édition n'est pas neutre, tant dans le choix des documents principaux ou annexés que dans leur présentation formelle, tant à cause de la réécriture des procèsverbaux par les assemblées successives (intense lors de la « réaction thermidorienne ») qu'eu égard à la formation des auteurs de cette somme.

(5) Colloque à Paris, les 20 et 21 septembre 2012. Michel BIARD, Philippe BouRdIN, Hervé LEUWERS, Pierre SERNA (dir.), 1792. Entrer en République, Paris, Armand Colin, 2013.

(6) Colloque à Tokyo, les 23 et 24 novembre 2013, et journée d'étude à Ivry-sur-Seine, le 7 novembre 2014. Actes à paraître : Michel BIARD, Philippe BOURDIN, Hervé LEUWERS, Yoshiaki ÔMI (dir.), L'écriture d'une expérience. Révolution, histoire et mémoires de conventionnels, Paris, SER, 2015.

(7) Colloque à Paris, les 18-20 septembre 2014. Actes à paraître : Michel BIARD, Philippe BOURDIN, Hervé LEUWERS, Alain TOURRET (dir.), Vertu et politique. Les pratiques des législateurs (1789-2014), Rennes, PUR, 2015. 
Les articles qui suivent empruntent, cette fois, deux des pistes de renouvellement que le programme ACTAPOL a voulu utiliser. La première est celle des hommes, des acteurs, déjà au cœur des travaux d'Alison Patrick ${ }^{8}$. Ici, le dépouillement des déclarations des revenus et du patrimoine des conventionnels encore en poste en vendémiaire an IV offre un matériau sans égal pour approcher les cultures professionnelles - toujours dominées par les juristes -, les compositions des fortunes, voire la vie familiale et matérielle, avec ses conséquences induites, jusqu'au déclassement social à l'issue d'un séjour parisien long et coûteux (Philippe Bourdin). Derrière les hommes, les « femmes de » transparaissent parfois, avant même que la Constitution de l'an III n'exige des Anciens qu'ils soient mariés. Jusqu'ici, elles ont peu retenu l'attention des historiens, à l'exception de quelques personnalités hors-norme. L'intérêt renouvelé pour les ego-documents, au-delà du domaine de l'affectif, permet de s'interroger sur la présence des femmes de conventionnels à Paris, sur leur sociabilité partagée dans un périmètre réduit autour de l'Assemblée, sur leur participation aux débats publics, sur l'ampleur et les formes de la solidarité obligée entre l'élu et son épouse volens nolens, celle-ci se trouve de fait impliquée par le comportement et les choix de son époux, comme celui-ci peut être amené à justifier des actions de sa femme, dans un entrelacs de plus en plus resserré entre vertu publique et vertus privées (Anne Jollet). À l'issue de leur engagement politique, les conventionnels demeurent ces hommes qui ont fait entrer la France en République, ont jugé le roi, ont agi ou ont vécu sous « la Terreur »; à l'heure de la seconde restauration des Bourbons, passés les ralliements aux Cent-Jours, le regard sur leur parcours reste éminemment politique, comme en témoignent ces notices historiques que le ministère de la Police générale, guidé par une peur paranoïaque du complot, fait rédiger sur les régicides vivants ou... morts. Proscrits ou non par la loi d'amnistie du 12 janvier 1816, exilés ou isolés dans leur « pays » natal, protégés par un réseau d'interconnaissances, les anciens conventionnels et leurs proches demeurent sous l'œil d'une police ou de corps diplomatiques soupçonneux, comme « prisonniers de la politique », contraints d'écrire leur futur au passé (Côme Simien).

Derrière les hommes, ce sont aussi les mouvances politiques et les formes changeantes de l'action qui se laissent deviner. Pour en renouveler l'approche, jusqu'alors solidement fondée sur l'étude sociologique des élus, sur l'analyse de leurs programmes et de leurs votes, s'impose

(8) Alison Patrick, The Men of the First French Republic. Political Alignments in the National Convention of 1792, Baltimore \& London, The Johns Hopkins University Press, 1972. 
une attention forte aux catégories léguées par les représentants ou, plus souvent, par l'historiographie qui les a forgées ou figées au XIX $\mathrm{X}^{\mathrm{e}}$ siècle. De la même manière qu'une compréhension renouvelée de « la Terreur » impose d'historiciser le mot qui la désigne ${ }^{9}$, le jeu des acteurs incite à continuer à s'interroger sur les catégories « Montagnard », « Girondin », « Brissotin », voire « sans-culotte ». D’importants corpora documentaires permettent d'examiner avec plus de détails la chronologie des emplois et les usages différenciés de ces notions et, partant, d'isoler les spécificités des langages politiques, dans le prolongement des volumes pionniers du Dictionnaire des usages socio-politiques (Marco Marin). Avec des sources différentes, et à partir de la seule notion de « Montagne », l'étude d'Alexandre Guermazi s'inscrit dans une perspective proche et rappelle les liens entre les mots et l'élaboration de projets politiques relevant d'une ambition d'organisation de l'espace public. Elle rappelle aussi la porosité entre les espaces tribuniciens - ceux des Jacobins et de la Convention -, et les exigences pédagogiques et prosélytes qui marquent les rapports entre les députés et leurs électeurs (ou les délégations de fédérés) dans le processus d'exclusion des girondins du cœur du pouvoir.

C'est alors l'action des représentants du peuple, dans et hors de l'enceinte des débats, qu'il s'agit de scruter. À la Convention, les échanges sont arbitrés par un président aux pouvoirs larges, mais aux fonctions difficiles et étroitement limitées dans le temps : primus inter pares, il distribue et gère la parole, maintient l'ordre dans la salle et les tribunes, représente l'institution dans les cérémonies officielles ou lors des accueils solennels, accorde des avis privés, dispose selon les moments de pouvoirs de nomination, etc. ; autour de lui, symboliquement placé en position élevée, se réunissent «droite » et « gauche ». S'interroger sur les quatrevingt-onze hommes qui ont exercé ce rôle (48\% sont des montagnards), sur leur âge et leur expérience, sur leur profil social et politique, sur le cumul de leurs responsabilités (participation aux comités, aux missions), ou sur leur manière de diriger les débats, de s'imposer, parfois en usant du geste plus que de la parole (se couvrant pour intimer le calme), c'est approcher d'une autre manière la vie publique, les divisions de l'Assemblée ou la question de la neutralité (Vincent Cuvilliers, Philippe Moulis, Matthieu Fontaine). Les relations des conventionnels avec leurs commettants, qui s'inscrivent depuis 1789 dans une stratégie d'échanges structurant la vie politique et

(9) Voir Michel BIARD, Hervé LEUWERS (dir.), Visages de la Terreur. L'exception politique de l'an II. Paris, Armand Colin, 2014, p. 5-14. 
en nationalisant les enjeux, peuvent être analysées par les correspondances publiques ou privées conservées. Ainsi celles des élus du Finistère, dont les lettres permettent de mieux comprendre les relations Paris-province au cœur même des événements de l'an II ; des relations qui, loin d'imposer à un département le rythme et les mots d'ordre politiques de Paris, révèlent les échanges réciproques entre des élus et leurs électeurs, les choix faits par les premiers de privilégier le récit des événements fédérateurs (les grandes fêtes parisiennes plutôt que l'exécution du roi, dont ils ont pourtant relaté le procès), leur souci d'exemplarité, leur difficulté à afficher des appartenances que leur républicanisme paraît subsumer, leurs divisions devant le mouvement populaire (Anne de Mathan). Dans une perspective qui rencontre les récentes publications de Michel Biard, Marisa Linton ou Hervé Leuwers ${ }^{10}$, Mette Harder s'interroge sur les raisons et la signification de cette permanence du rejet de l'adversaire politique, de part et d'autre de la rupture de Thermidor, en relativisant les explications par les spécificités politiques de l'an II ; n'y aurait-il pas des traits communs aux épurations de la Convention montagnarde et de la Convention thermidorienne ?

Les constituants avaient eu un « Nouveau Régime » à penser et à mettre en œuvre; les membres de la Convention nationale eurent, quant à eux, une République à fonder dans un contexte pour le moins extraordinaire, les années 1792-1795 devenant un moment clé de la Révolution. Redécouvrir les itinéraires individuels permet à coup sûr de repenser les origines de la Première République, mais aussi toute une culture de l'affrontement politique et les conditions dans lesquelles les conventionnels en vinrent à la décision de proroger leurs pouvoirs en 1795,

(10) Michel BIARD, La Liberté ou la mort. Mourir en député, 1792-1795, Paris, Tallandier, 2015 ; Marisa Linton, Choosing Terror. Virtue, Friendship, and Authenticity in the French Revolution, Oxford, Oxford University Press, 2013 ; Hervé LEUWERS, Robespierre, Paris, Fayard, 2014. 
ces deux derniers points étant évidemment fondamentaux pour les héritages légués aux siècles suivants.

Michel BIARD

GRHis, université de Rouen

61 rue lord Kitchener, 76600 le Havre

biard.m@wanadoo.fr

Philippe BOURDIN

CHEC, université Clermont-Ferrand 2 103 bd Lafayette, 63000 Clermont-Ferrand philippe.bordin@laposte.net

Hervé LEUWERS

IRHiS, université Lille 3

4 Grande Voie, 62173 Rivière herve.leuwers@laposte.net 